\title{
Nitrogen swirl: Creating rotating polygons in a boiling liquid
}

\author{
Alexis Duchesne $\odot^{*}$ and Tomas Bohr ${ }^{\dagger}$ \\ Department of Physics, Technical University of Denmark, DK-2800 Kgs. Lyngby, Denmark \\ Barbara Bohr \\ Asger Rygsgade, DK-1727, Copenhagen V, Denmark \\ Laust Tophøj \\ TG Dampers ApS, DK-4700 Naestved, Denmark
}

(Received 30 January 2019; published 24 October 2019)

\begin{abstract}
This paper is associated with a video winner of a 2018 APS/DFD Gallery of Fluid Motion Award for work presented at the DFD Gallery of Fluid Motion. The original video is available online at the Gallery of Fluid Motion, https://doi.org/10.1103/APS.DFD.2018. GFM.V0032.
\end{abstract}

DOI: 10.1103/PhysRevFluids.4.100507

When we learn about rotating flows, we usually start with the most famous example: Newton's rotating bucket. Newton attached a bucket of water to the ceiling by a rope and twisted the rope. As he let go, the bucket started rotating, and after a while the water came to rest again. This time, however, the water surface was no longer flat but curved upward towards the rim like a paraboloid, showing that the rest frame of the rotating bucket is not an inertial frame. In the video presented here, we perform an experiment which is a variation on that theme. Here we stir a volume of liquid with very low viscosity in a stationary pot and watch it while the swirling motion gradually decays. Surprisingly, this happens through a sequence of quasistationary states with polygonal surface deformations, as described in Ref. [1].

In the experiment, between 0.5 and 21 of liquid nitrogen is poured into a stationary pot maintained at a temperature of $T_{P}=353 \mathrm{~K}\left(80^{\circ} \mathrm{C}\right)$ in a water bath. This liquid has a viscosity five times smaller than water and a boiling temperature $T_{N}=77 \mathrm{~K}\left(-196^{\circ} \mathrm{C}\right)$, which means that it undergoes "film boiling." Thus, the liquid is separated from the solid surfaces by a thin vapor layer and is held in a "Leidenfrost" state with extremely low friction. The ensuing film boiling also insulates the liquid, so that it takes several minutes before the whole volume of liquid has evaporated.

Then the liquid is set into rotation by stirring manually with a wooden spoon. We stir the liquid so vigorously that it is flung away from the central part of the pot, which then becomes dry while the surface rises at the sides of the pot. The surface rapidly acquires a characteristic convex shape, running from the central dry circle on the bottom of the pot to the liquid interface at the sidewall, as

\footnotetext{
*alexis.duchesne@univ-lille.fr

†tbohr@fysik.dtu.dk
}

Published by the American Physical Society under the terms of the Creative Commons Attribution 4.0 International license. Further distribution of this work must maintain attribution to the author(s) and the published article's title, journal citation, and DOI. 

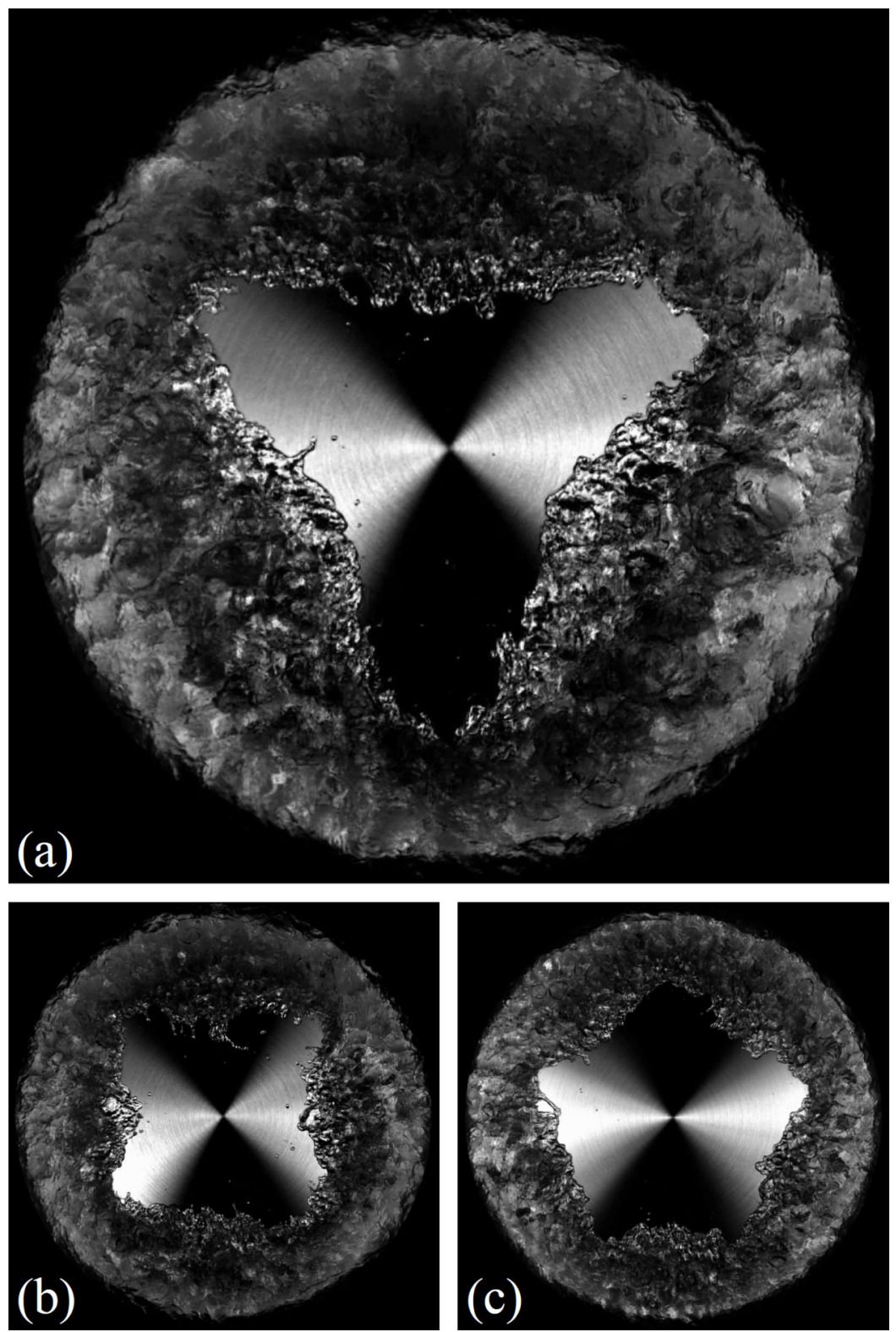

FIG. 1. Rotating polygons in liquid nitrogen rotating flow. Liquid nitrogen (boiling temperature $T_{N}=77 \mathrm{~K}$, viscosity $v=2 \times 10^{-7} \mathrm{~m}^{2} \mathrm{~s}^{-1}$ and density $\rho=810 \mathrm{~kg} \mathrm{~m}^{-3}$ ) is poured into a stationary pot, placed in a water bath kept at $80^{\circ} \mathrm{C}$. The boiling liquid is set into rotation using a wooden spoon, and then the pot is covered with a transparent glass, leaving a small opening allowing the nitrogen vapor to push out the aqueous mist that obstructs the view. From above, one can observe "polygons" with two to six corners, e.g., triangles (a), squares (b), and pentagons (c). As the fluid evaporates and slows down, the number of corners gradually decreases. DOI: 10.1103/APS.DFD.2018.GFM.V0032.

opposed to the concave surface shape of Newton's bucket. The azimuthal flow speed varies like $1 / r$ (like a line vortex) and thus becomes more rapid closer to the axis. The reason is the "frustrated" character of the nitrogen flow: since the pot is standing still, the flow is gradually slowing down, 
and this invariably induces a secondary radial flow on top of the dominant azimuthal one, turning it into something like a line vortex flow. Then, on a somewhat longer timescale, symmetry-breaking instabilities set in, they deform the surface into polygonal structures with somewhere between two and six corners, starting at some value depending on the liquid volume and speed and then slowly removing the corners one by one. For a given volume of liquid, the larger the rotation speed, the larger the number of initial corners, and, conversely, for a given rotation speed, the smaller the liquid volume, the larger the number of corners. However, we have never observed polygons with more than six corners.

We believe that this instability leading to the polygons is caused by the resonance between "gravity waves" (living near the almost horizontal surface at the rim) and "centrifugal waves" (living near the strongly inclined surface at the center) as developed in Refs. [1,2]. Interestingly, it is the resonance between a forward-moving gravitational wave and a backward-moving centrifugal wave which creates the instability. For this to be possible, the "backward" centrifugal wave must actually move forward in the laboratory frame, and thus the flow speed near the center has to be greater than that of the wave (corner), making this part of the flow "supercritical"-something one can easily verify by looking at the video. As can be seen from Fig. 1, the flow is strongly turbulent, something which is not included in the model. It is surprising that such orderly coherent states can survive and remain in synchrony despite the chaotic bursts and eruptive drop detachments seen in the video. Actually, we believe that these chaotic bursts enhance the exchange of angular momentum between different parts, thereby facilitating the approach to the constant-angular-momentum line vortex flow, from which the instability can proceed. This phenomenon therefore illustrates the ability of turbulent rotating flows to generate order and is reminiscent of such large-scale planetary phenomena as Jupiter's Red Spot and Saturn's North Pole hexagon.

The research leading to these results has received funding from the People Programme (Marie Curie Actions) of the European Union's Seventh Framework Programme (FP7/2007-2013) under REA Grant Agreement No. 609405 (COFUND Postdoc DTU). We are grateful to Erik Hansen, Jacob Søberg Bach, Mette Høst, Asbjørn Lauritsen, and Martijn van der Ouderaa for helpful advice and ideas on the setup.

[1] L. Tophøj, J. Mougel, T. Bohr, and D. Fabre, Rotating Polygon Instability of a Swirling Free Surface Flow, Phys. Rev. Lett. 110, 194502 (2013).

[2] J. Mougel, D. Fabre, L. Lacaze, and T. Bohr, On the instabilities of a potential vortex with a free surface, J. Fluid Mech. 824, 230 (2017). 